\title{
INTER-DIVISION IV-V-IX / WORKING GROUP STANDARD STARS
}

\author{
CHAIR \\ NEWSLETTER EDITOR
}

\author{
Christopher J. Corbally \\ Richard O. Gray
}

TRIENNIAL REPORT 2006 - 2009

\section{Introduction}

The Working Group, created in 1982 during the IAU General Assembly in Patras (Greece), exists to examine, organize, and coordinate the various sets of standard stars for the fields in which they are used: radial velocities, spectral classification, photometry, astrometry, and others. It also provides a forum for discussion and education. All those working with and upon standard stars are considered members of the WG.

During 2007 the WG, with the support of Monique Spite, the Division IV president of its hosting Commission 45 on Stellar Classification, was changed to an Inter-Division Working Group. This was done since it was realized that some of the original Commissions from which the WG was formed now belonged to different Divisions. These are Commissions 25 on Stellar Photometry and Polarimetry, 29 on Stellar Spectra, and 30 on (Radial Velocities, to all of which standard star issues are essential. Hence the WG now spans Divisions IV, V, and IX.

\section{Activities}

As its main activity the WG publishes The Standard Star Newsletter in April and October of each year. This has contained research notes, abstracts of papers, websites of interest, and reports and announcements of meetings, all with relevance to standard stars and their use in astronomy. These newsletters can be downloaded from <stellar.phys.appstate.edu/ssn/>.

Other activities in this period have included: work on a spectral-type coding system, spanning the range of standard and peculiar stars, which will be incorporated into MAST's (Multi-Mission Archive at Space Telescope) search engine; advice to the U.S. Naval Observatory on incorporation of spectrophotometric standards into the Astronomical Almanac; and discussion of a 'Standard Field' in which different surveys could make direct comparisons of their calibrations for stellar data.

Papers from the meeting on Future of Photometric, Spectrophotometric and Polarimetric Standardization have been published in the ASP Conference Series 2007 under the editorship of Christiaan Sterken.

With sadness I report the passing of a founding member of the WG and the first editor of The Standard Star Newsletter, Laura E. Pasinetti, on 12 September 2006.

Christopher J. Corbally chair of the Working Group 